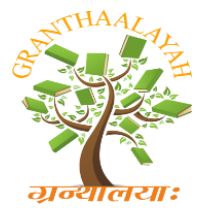

INTERNATIONAL JOURNAL OF RESEARCH GRANTHAALAYAH

A knowledge Repository

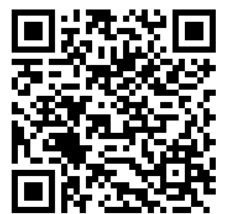

Science

\title{
ELECTRO OPTIC MODULATOR DEVICES FOR HIGH SPEED DATA IN OPTICAL COMMUNICATION
}

\author{
Raghunathababu.CH*1, Dr. Siddaiah.P2 \\ ${ }^{* 1}$ Junior Research Fellow, ECE Department, Acharya Nagarjuna University, Guntur, A.P., \\ INDIA \\ ${ }^{2}$ Principal, Acharya Nagarjuna University, Guntur, A.P., INDIA
}

\begin{abstract}
Electro Optic devices in fiber optic Communication systems are playing a Vital Role in telecommunication infrastructure for worldwide broadband networks. Wide bandwidth of signal transmission with low delay, wide transmission bandwidth with less latency, the transmission medium for long distance with high data rate transmission. This paper gives an overview of Electro optic devices for fiber optic systems and their key technologies, their technological trend towards the next generation.
\end{abstract}

Keywords:

Electro optic modulator, $3 d$ b values, irradiation values, LiNbO3.

Cite This Article: Raghunathababu.CH, and Dr. Siddaiah.P, "ELECTRO OPTIC MODULATOR DEVICES FOR HIGH SPEED DATA IN OPTICAL COMMUNICATION" International Journal of Research - Granthaalayah, Vol. 3, No. 10(2015): 38-42.

\section{INTRODUCTION}

The carrier concentration in silicon can be varied by injection, accumulation, depletion, or inversion of carriers. The PIN diodes and Metal Oxide Semiconductor Field Effect Transistors (MOSFET) are employed for this purpose. In MOS devices, the carriers are typically located only in a small region, resulting in a small overlap between the optical mode and the non-equilibrium charge distribution in the waveguide, and thereby leading to a smaller effective index variation in a MOS system than that in a PIN configuration [1]. A small effective index change requires a very long structure, of the order of milli meters, in order to induce a significant modulation depth. Super High Frequencies are using for Microwave devices, Modern RADAR, Communication Devices, and Digital Video Broadcasting (DVBS) etc. There are many Electro Optical (EO), Electronic Warfare (EW) Systems which are analogous to Radio Frequency (RF) EW systems. These EO EW systems operate in the optical portion of the electromagnetic spectrum. Electro optics (EO), as the name implies, is a combination of electronics and optics. By one definition EO is the science and technology of the generation, modulation, detection and measurement, or display of optical radiation by electrical means most infrared (IR) sensors. 


\section{RESULTS AND DISCUSSIONS}

\section{A. Electro optics}

The Electro Optic effect is the change in the refractive index resulting from the application of a DC or low frequency electric field. A field applied to an anisotropic Electro Optic material modifies its refractive indices and thereby its effect on polarized light.

\section{B. Modulators}

Encode analog or digital signals on an optical wave. Shift optical power to other spectral frequency bands. Control power and or phase of a beam. Act as fast switches for laser Q switching, etc. At present, we have taken one of the most important components of a nano photonic communication system is a fast electro optic modulator [2], which takes in a direct current optical input signal and switches it on/off using a high data rate electronic signal. Modulation is achieved by inducing a change in the phase or the intensity of the light, using a refractive index change or an absorption change, respectively. Moreover we have analyzed parametrically the transmission data rate, operating signal bandwidth, modulation bandwidth, device performance index, and transmission data rate length product within NRZ (Non Return to Zero)coding as a good criteria for high speed electro optic device performance and efficiency.

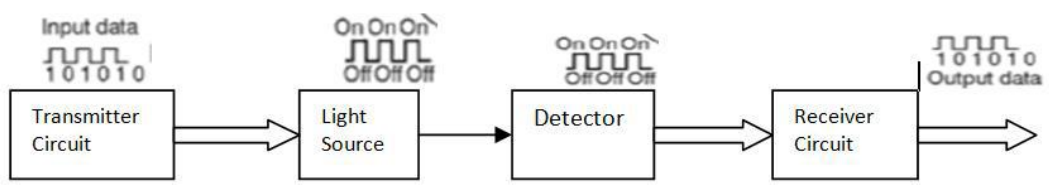

Figure 1: Basic Fiber Optical Communication System.

The Fig.1shows the Basic Fiber Optical Communication system and each stage show its wave form Representation. The Below Graph Representing the Modulator Devices Sensitivity how it reduce with Irradiation Values shown in Fig.2.

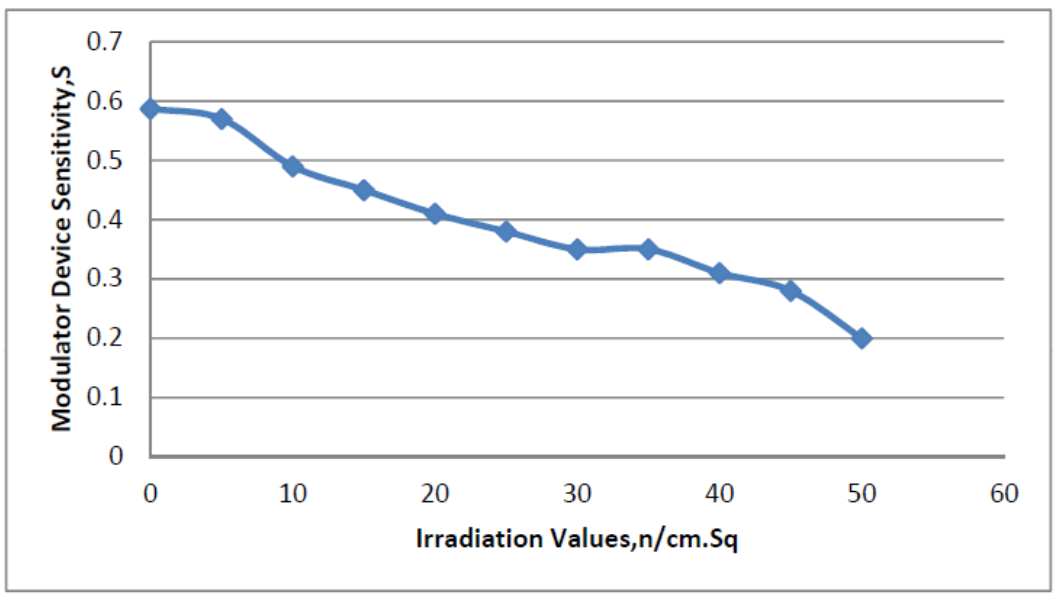

Figure 2: Variation of Electro Optic Modulator Devices Sensitivity Reduces with Irradiation Values. 
The below Fig.3 Representing the Variation of 3dB Signal Transmitted Bandwidth Reduces with Irradiation Assumed Values. Here power absorption coefficient and modulator length increase, it results in decreasing of modulation device bandwidth. So possible to enhance the bandwidth by this Experiment.

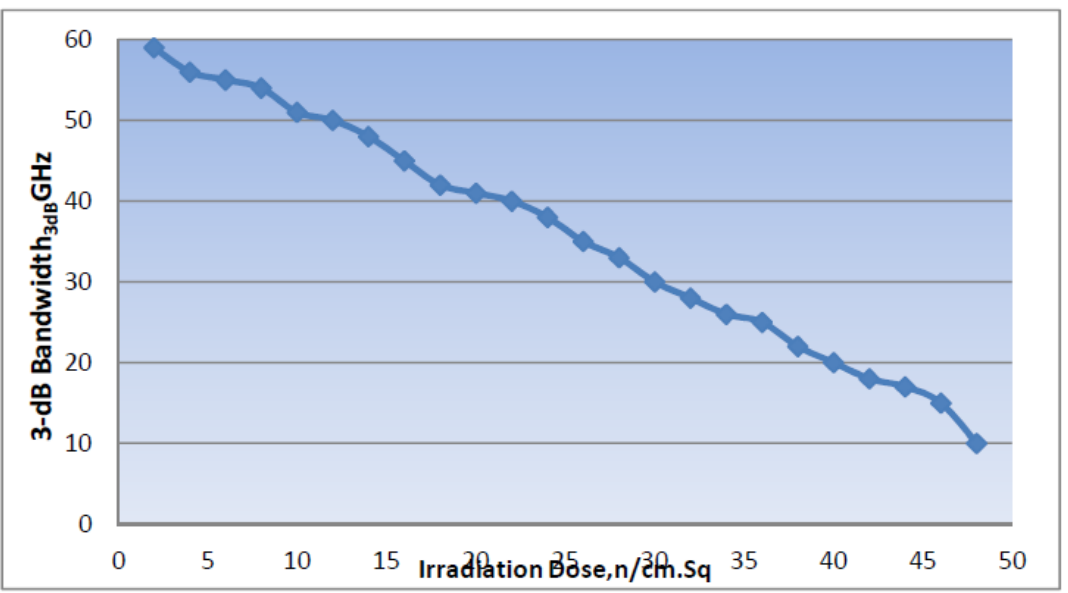

Figure 3: Variation of 3dB Signal Transmitted Bandwidth Reduces with Irradiation Assumed Values.

From Fig.4.modulator length and relative refractive index difference increase, this leads to increase in modulating voltage, and operating optical signal wavelength increases then increasing of switching voltage possible to increase the data rate.

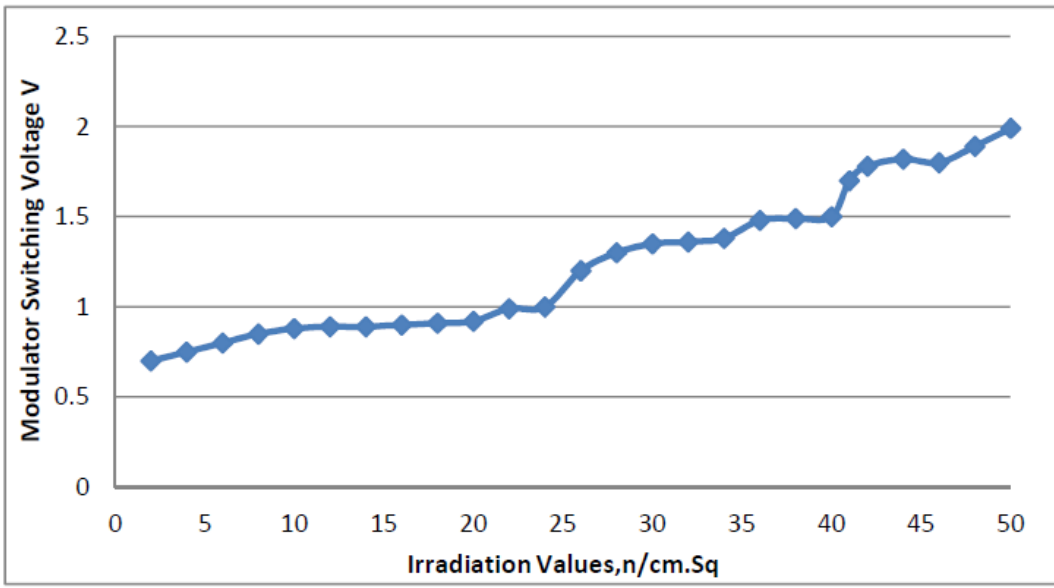

Figure 4: Variation of Modulator Switching Voltage Increases with Irradiation Assumed set of parameters.

\section{Advantages of Integrated Optic Modulators}

Integrated optic modulators require lower drive voltages and operate at higher frequencies than bulk modulators. Because of their small size and compatibility with single mode optical fiber, they have been used in a variety of communication and sensor applications. Phase modulators have been used in digital optical communication experiments using 4Gbps phase shift keyed (PSK) modulation, 4Gbps differential phase shift keyed (DPSK) modulation and 8Gbps quadrature phase shift keyed (QPSK) modulation [4]. Amplitude modulators are used in a $770 \mathrm{~km}, 2.5 \mathrm{Gbps}$ long 
distance optical communication, a $20 \mathrm{GHz}$ analog link, a 50 channel AM video transmission demonstration. Modulators with both phase and amplitude sections integrated on the same chip have been used to transmit $125 \mathrm{Mbps}$ amplitude shift-keyed (ASK) data and 2.488 Gbps PSK data simultaneously on the same light wave.

The ferroelectric material lithium niobate (LiNbO3) [5] has been applied to optical devices. It has excellent electro optic and optical properties are large electro optic effect and capable of a highspeed response. It is also transparent for infrared light and easy to fabricate into low loss channel waveguides by diffusing titanium. The electro absorption in the devices tested was tuned to obtain a maximum modulation depth at a wavelength of $1.54 \mu \mathrm{m}$. Reflectivity of the device is modulated by varying the applied electric field across the quantum wells [3].The polarization reversed structure is applicable to several EO optical modulators based on ferroelectric materials (LiNbO3, $\mathrm{Li} \mathrm{TaO} 3, \mathrm{KNbO} 3$, etc.), and it enables us to construct novel devices with advanced functions and performances. Now Government of India establishing 100 Smart cities over Country wide and more satellite townships. Aim of this project is provide High speed data to Common people to access all the government schemes.

\section{Units}

In this paper all the Represented units are international standards. Used units are $\mathbf{n} / \mathbf{c m} . \mathbf{s q}$ for Newton per square Centimeter and $\mathbf{V}$ for Voltage.

\section{RESULTS}

In a summary, presentation of high speed semiconductor electro optic modulator devices for multi gigahertz operation systems. Theoretically found that, increased confinement factor and modulator length, and the decreased operating optical signal wavelength, this leads to the decreased switching device voltage. It is evident that the decreased operating optical signal wavelength and modulator thickness, increased confinement factor, results in increasing of device performance index. Similarly, the increased output received power and modulator length, and the increased operating optical signal wavelength, and the decreased ambient temperature, this result in the increased transmission bit rate.

\section{CONCLUSIONS}

There is a need of Research work to be develop the faster data rates with current and future data traffic, advanced switching techniques and more intelligent network architectures. This practical deployment thereby leading to a new generation in fiber optics communications.

\section{ACKNOWLEDGMENT}

I am very grateful and would like to thank Dr. Siddaiah, $\mathrm{PhD}$. advices, instructions and continued support without him it would not have been possible for me to complete this report.

Also would like to thank all my friends and colleague for all the thoughtful and mind stimulating discussions had, which prompted me to think beyond the obvious. 


\section{REFERENCES}

[1] Francis Idachaba, Dike U.Ike, and Orovwode Hope, "Future Trends in Fiber Optic Communication," Proceedings of the World Congress on Engineering, July 2 - 4, 2014, London, U.K.

[2] Eng. ISSA OBAID ALORF, "The Recent Trends in Fibre Optic Communication”, IOSR Journal of Electrical and Electronics Engineering, Apr. 2014.

[3] "Recent Trends and Developments in optical Communication", Unger, H.G, Hochfreqenztechnik, TU Braunschweig, Microwave Conference, $1985.15^{\text {th }}$ European,published by IEEE.

[4] Lee, Cheng Chung (Ed.), "The Current Trends in Optics and Photonics," by springer publications 2015.

[5] "Current Research and Development In Optical Fiber Communications In China," by Q.M. Wang and T. P. Lee, Published by World Scientific Ltd 1996. 